\title{
Intracluster Planetary Nebulae as dynamical probes of the diffuse light in galaxy clusters
}

\author{
Magda Arnaboldi
}

INAF, Osservatorio Astronomico di Torino, Strada Osservatorio, 20, I-10025 Pino Torinese

\begin{abstract}
I will review the latest results for the presence of diffuse light in the nearby universe and at intermediate redshift, and then discuss the latest results from hydrodynamical cosmological simulations of cluster formation on the expected properties of diffuse light in clusters. I shall present how intracluster planetary nebulae (ICPNe) can be used as excellent tracers of the diffuse stellar population in nearby clusters, and how their number density profile and radial velocity distribution can provide an observational test for models of cluster formation. The preliminary comparison of available ICPN samples with predictions from cosmological simulations support late infall as the most likely mechanism for the origin of diffuse stellar light in clusters.
\end{abstract}

\section{OBSERVATIONS OF DIFFUSE LIGHT}

The study of the intracluster light (ICL) began with Zwicky's (1951) claimed discovery of an excess of light between galaxies in the Coma Cluster. Its low surface brightness $\left(\mu_{B}>28 \mathrm{mag} \operatorname{arcsec}^{-2}\right)$ makes it difficult to study the ICL systematically (Oemler 1973; Thuan \& Kormendy 1977; Bernstein et al. 1995; Gregg \& West 1998; Gonzalez et al. 2000).

Presence of diffuse light can be revealed by tails, arcs or plumes which are narrow (about $\sim 2 \mathrm{kpc}$ ) and extended $(\sim 50-100 \mathrm{kpc})$, or as a halo of light at the cluster scales, which is present in the $200-700 \mathrm{kpc}$ radial range. In the Coma cluster, Adami et al. (2005) have searched for ICL small features using a wavelet analysis and reconstruction technique. They identified 4 extended sources, with 50 to $100 \mathrm{kpc}$ diameter and V band magnitudes in the 14.5 - 16.0 range. Quantitative, large scale measurements of the diffuse light in the Virgo cluster were recently attempted by Mihos et al. (2005): these deep observations show the intricate and complex structure of the ICL in Virgo.

On the cluster scales, the presence of diffuse light can be revealed when the whole distribution of stars in clusters is analysed in a way similar to Schombert's (1986) photometry of brightest cluster galaxies (BCGs). When this component is present, the surface-brightness profiles centred on the BCG turn strongly upward in a $\left(\mu, R^{1 / \alpha}\right)$ plot for radii from 200 to $700 \mathrm{kpc}$. This approach to ICL low surface brightness measurements was taken by $\mathrm{Zi}$ betti et al. (2005), who studied the spatial distribution and colors of the ICL in 683 clusters of galaxies at $z \simeq 0.25$ by stacking their images, after rescaling them to the same metric size and masking out resolved sources.

In nearby galaxy clusters, intracluster planetary nebulae (ICPNe) can be used as tracers of the ICL; this has the advantages that detection of ICPNe are possible with deep narrow band images and that the ICPN radial velocities can be measured to investigate the dynamics of the ICL component. ICPN candidates have been identified in Virgo (Arnaboldi et al. 1996, 2002, 2003; Feldmeier et al. 2003, 2004a) and Fornax (Theuns \& Warren 1997), with significant numbers of ICPN velocities beginning to become available (Arnaboldi et al. 2004).

The overall amount of the ICL in galaxy clusters is still a matter of debate. However, there is now observational evidence that it may depend on the physical parameters of clusters, with rich galaxy clusters containing $20 \%$ or more of their stars in the intracluster component (Gonzalez et al. 2000; Gal-Yam et al. 2003), while the Virgo Cluster has a fraction of $\sim 10 \%$ in the ICL (Ferguson et al. 1998; Durrell et al. 2002; Arnaboldi et al. 2002, 2003; Feldmeier et al. 2004a), and the fraction of detected intragroup light (IGL) is $1.3 \%$ in the M81 group (Feldmeier et al. 2004b) and less than $1.6 \%$ in the Leo I group (Castro-Rodríguez et al. 2003). Recent hydrodynamical simulations of galaxy cluster formation in a $\Lambda \mathrm{CDM}$ cosmology have corroborated this observational evidence: in these simulated clusters, the fraction of the ICL increases from $\sim 10 \%-20 \%$ in clusters with $10^{14} M_{\odot}$ to up to $50 \%$ for very massive clusters with $10^{15} M_{\odot}$ (Murante et al. 2004). Strong correlation between ICL fraction and cluster mass is also predicted from semi-analytical models of structure formation (Lin \& Mohr 2004).

The mass fraction and physical properties of the ICL and their dependence on cluster mass will be related with 
the mechanisms by which the ICL is formed. Theoretical studies predict that if most of the ICL is removed from galaxies because of their interaction with the galaxy cluster potential or in fast encounters with other galaxies, the amount of the ICL should be a function of the galaxy number density (Richstone \& Malumuth 1983; Moore et al. 1996). The early theoretical studies about the origin and evolution of the ICL suggested that it might account for between $10 \%$ and $70 \%$ of the total cluster luminosity (Richstone \& Malumuth 1983; Malumuth \& Richstone 1984; Miller 1983; Merritt 1983, 1984). These studies were based on analytic estimates of tidal stripping or simulations of individual galaxies orbiting in a smooth gravitational potential. Nowadays, cosmological simulations allow us to study in detail the evolution of galaxies in cluster environments (see, e.g., Moore et al. 1996; Dubinski 1998; Murante et al. 2004; Willman et al. 2004; Sommer-Larsen et al. 2005). Napolitano et al. (2003) investigated the ICL for a Virgo-like cluster in one of these hierarchical simulations, predicting that the ICL in such clusters should be unrelaxed in velocity space and show significant substructures; spatial substructures have been observed in one field in the ICPNe identified with [O III] and $\mathrm{H} \alpha$ (Okamura et al. 2002).

\section{DIFFUSE LIGHT IN CLUSTERS FROM COSMOLOGICAL SIMULATIONS}

Cosmological simulations of structure formation facilitate studies of the diffuse light and its expected properties. Dubinski (1998) constructed compound models of disk galaxies and placed them into a partially evolved simulation of cluster formation, allowing an evolutionary study of the dark matter and stellar components independently. Using an empirical method to identify stellar tracer particles in high-resolution cold dark matter (CDM) simulations, Napolitano et al. (2003) studied a Virgo-like cluster, finding evidence of a young dynamical age of the intracluster component. The main limitations in these approaches is the restriction to collisionless dynamics.

Murante et al. (2004) analyzed for the first time the ICL formed in a cosmological hydrodynamical simulation including a self-consistent model for star formation. In this method, no assumptions about the structural properties of the forming galaxies need to be made, and the gradual formation process of the stars, as well as their subsequent dynamical evolution in the non-linearly evolving gravitational potential can be seen as a direct consequence of the $\Lambda \mathrm{CDM}$ initial conditions.

Murante et al. (2004) identified 117 clusters in a large volume of $192^{3} h^{-3} \mathrm{Mpc}^{3}$, and analyze the correlations of properties of diffuse light with, e.g., cluster mass and
X-ray temperatures. Galaxies at the centers of these clusters have surface-brightness profiles which turn strongly upward in a $\left(\mu, R^{1 / \alpha}\right)$ plot. This light excess can be explained as IC stars orbiting in the cluster potential. Integrating its density distribution along the line-of-sight (LOS), the slopes from Murante et al. (2004) simulations are in agreement with those observed for the surface brightness profiles of the diffuse light in nearby clusters.

At large cluster radii, the surface brightness profile of the ICL appears more centrally concentrated than the surface brightness profile of cluster galaxies (see Figure 1). The prediction of ICL being more centrally concentrated than the galaxy cluster light has been tested observationally. Zibetti et al. (2005) have presented surface photometry from the stacking of 683 clusters of galaxies imaged in the g-, r-, and i-bands in the SDSS. They have been able to measure surface brightness as deep as $\mu_{r} \sim 32$ mag $\operatorname{arcsec}^{-2}$ for the ICL light and $\mu_{r} \sim 29.0$ for the total light, out to $700 \mathrm{kpc}$ from the BCG. They finds that the ICL is significantly more concentrated than the total light.

From the simulations carried out by Murante et al. (2004), they also obtained the redshifts $z_{\text {form }}$ at which the stars formed: those in the IC component have a $z_{\text {form }}$ distribution which differs from that in cluster galaxies, see Figure 2 The "unbound" stars are formed earlier than the stars in galaxies. The prediction for an old stars' age in the diffuse component agrees with the HST observation of the IRGB stars in the Virgo IC field, e.g. $t>2$ Gyr (Durrell et al. 2002), and points toward the early tidal interactions as the preferred formation process for the ICL. The different age and spatial distribution of the stars in the diffuse component indicate that it is a stellar population that is not a random sampling of the stellar populations in cluster galaxies.

Murante et al. (2004) studied the correlation between the fraction of stellar mass in the diffuse component and the clusters' total mass in stars, based on their statistical sample of 117 clusters. This fraction is $\sim 0.1$ for cluster masses $M>10^{14} h^{-1} M_{\odot}$ and it increases with cluster mass: the more massive clusters have the largest fraction of diffuse light, see Figure 2]. For $M \sim 10^{15} h^{-1} M_{\odot}$, simulations predict as many stars in the diffuse component as in cluster galaxies.

\section{Predicted dynamics of the ICL}

In the currently favored hierarchical clustering scenario, fast encounters and tidal interactions within the cluster potential are the main players of the morphological evolution of galaxies in clusters. Fast encounters and tidal stirring cause a significant fraction of the stellar component in individual galaxies to be stripped and dis- 


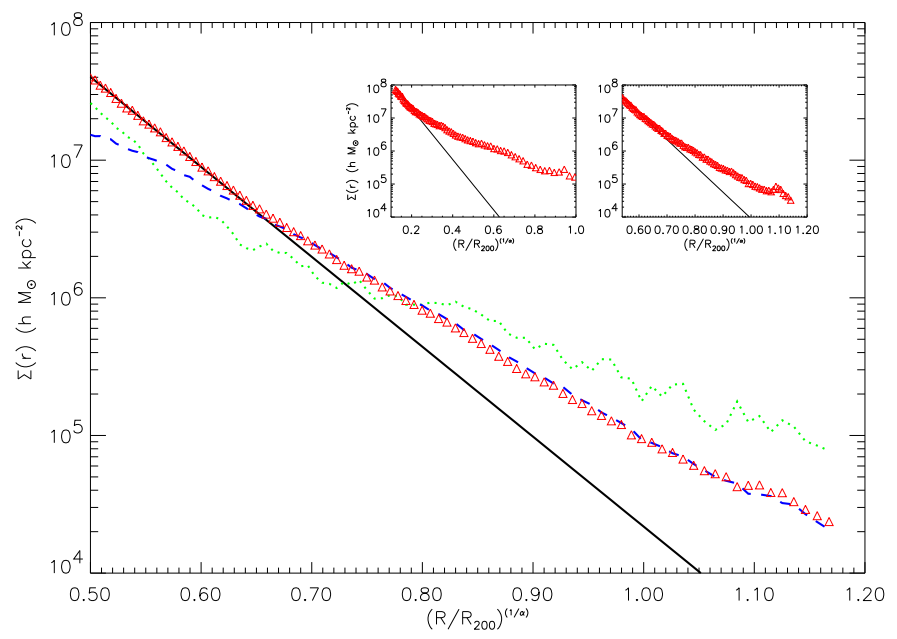

FIGURE 1. Schombert-like analysis on the stacked 2D radial density profile (BCG + ICL) of clusters in the Murante et al (2004) simulation (triangles). The light excess is evident at large cluster radii. The solid line shows the function $\log \Sigma(r)=$ $\log \Sigma_{e}-3.33\left[\left(r / r_{e}\right)^{1 / \alpha}-1\right]$, with best-fit parameters $\log \Sigma_{e}=20.80, r_{e}=0.005, \alpha=3.66$ to the BCG inner stellar light. Also shown are the averaged 2D density profile of stars in galaxies (dotted line) and in the field (dashed line). In the inserts, the results are shown from the same analysis for the most luminous clusters with $T>4 \mathrm{keV}$ (left panel), and for less luminous ones with $0<T<2 \mathrm{keV}$ (right panel). The resulting best-fit parameters are respectively $\log \Sigma_{e}=16.47, r_{e}=0.11, \alpha=1.24$ and $\log \Sigma_{e}=23.11, r_{e}=0.00076, \alpha=4.37$. In the main plot and in the inserts the unit $\left(R / R_{200}\right)^{1 / \alpha}$ refers to the $\alpha$ values given by each Sersic profile. From Murante et al. (2004).

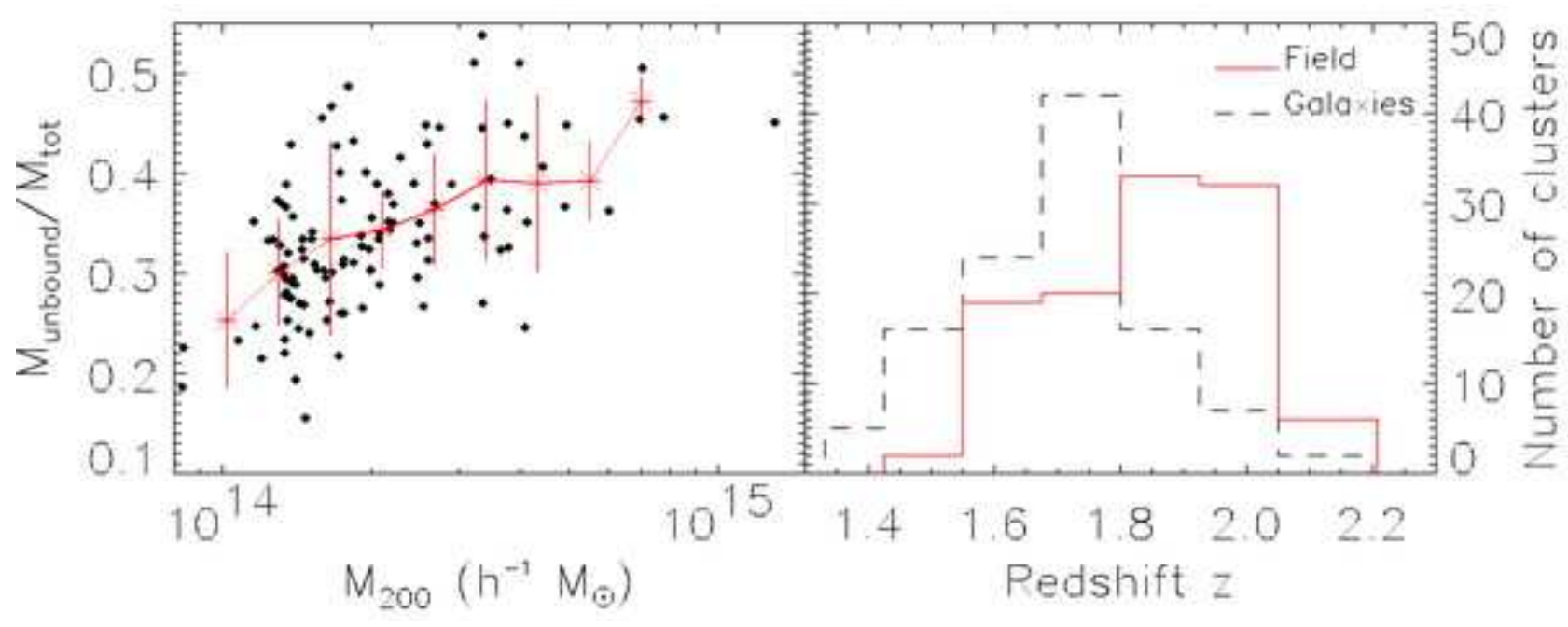

FIGURE 2. Left: Fraction of stellar mass in diffuse light vs. cluster mass. Dots are for clusters in the simulated volume; asterisks show the average values of this fraction in 9 mass bins with errorbars. Right panel: histograms of clusters over mean formation redshift, of their respective bound (dashed) and IC star particles (solid line). Mean formation redshifts are evaluated for each cluster as the average on the formation redshift of each star particle. From Murante et al. (2004). 


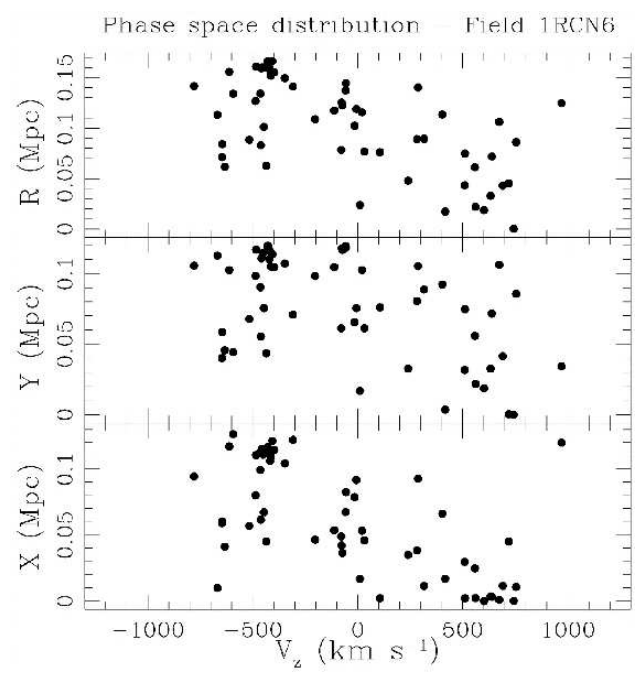

FIGURE 3. Projected phase-space diagram for a simulated ICPN sample in a N-body simulation of a Virgo-like cluster. From Napolitano et al. (2003).

persed within the cluster in a few dynamic times. If the timescale for significant phase-mixing is on the order of few cluster internal dynamical times, then a fraction of the ICL should still be located in long streams along the orbits of the parent galaxies. Detections of substructures in phase space would be a clear sign of late infall and harassment as the origin of the ICL.

A high resolution simulation of a Virgo-like cluster in a $\Lambda C D M$ cosmology was used to predict the velocity and the clustering properties of the diffuse stellar component in the intracluster region at the present epoch (Napolitano et al. 2003). The simulated cluster builds up hierarchically and tidal interactions between member galaxies and the cluster potential produce a diffuse stellar component free-flying in the intracluster medium. The simulations are able to predict the radial velocity distribution expected in spectroscopic follow-up surveys: they find that at $z=0$ the intracluster stellar light is mostly dynamically unmixed and clustered in structures on scales of about $50 \mathrm{kpc}$ at a radius of $400-500 \mathrm{kpc}$ from the cluster center.

Willman et al. (2004) and Sommer-Larsen et al. (2005) have studied the dynamics of the ICL in cosmological hydrodynamical simulations for the formation of a rich galaxy cluster. In a Coma-like rich cluster, Willman et al. (2004) finds that the ICL show significant substructure in velocity space, tracing separate streams of stripped IC stars. Evidence is given that despite an un-relaxed distribution, IC stars are useful mass tracers, when several fields at a range of radii have measured LOS velocities. According to Sommer-Larsen et al. (2005), IC stars are colder than cluster galaxies. This is to be expected because diffuse light is more centrally concentrated than cluster galaxies, as found in cosmological simulations (see Murante et al. 2004) and confirmed from observations of intermediate redshift clusters (Zibetti et al. 2005), and both the ICL and galaxies are in equilibrium with the same cluster potential.

\section{INTRACLUSTER PLANETARY NEBULAE IN THE VIRGO CLUSTER: THE PROJECTED PHASE SPACE DISTRIBUTION}

Intracluster planetary nebulae (ICPNe) have several unique features that make them ideal for probing the ICL. The diffuse envelope of a PN re-emits $15 \%$ of the UV light of the central star in one bright optical emission line, the green $[\mathrm{OIII}] \lambda 5007 \AA$ line. PNe can therefore readily be detected in external galaxies out to distances of $25 \mathrm{Mpc}$ and their velocities can be determined from moderate resolution $(\lambda / \Delta \lambda \sim 5000)$ spectra: this enables kinematical studies of the IC stellar population.

$\mathrm{PNe}$ trace stellar luminosity and therefore provide an estimate of the total IC light. Also, through the [OIII] $\lambda 5007 \AA$ planetary nebulae luminosity function (PNLF), $\mathrm{PNe}$ are good distance indicators, and the observed shape of the PNLF provides information on the LOS distribution of the IC starlight. Therefore ICPNe are useful tracers to study the spatial distribution, kinematics, and metallicity of the diffuse stellar population in nearby clusters.

\section{Current narrow band imaging surveys}

Several groups (Arnaboldi et al. 2002, 2003; Aguerri et al. 2005; Feldmeier et al. 2003, 2004a) have embarked on narrow-band [OIII] imaging surveys in the Virgo cluster, with the aim of determining the radial density profile of the diffuse light, and gaining information on the velocity distribution via subsequent spectroscopic observations of the obtained samples. Given the use of the PNLF as distance indicators, one also obtain valuable information on the 3D shape of the Virgo cluster from these ICPN samples.

Wide-field mosaic cameras, such as the WFI on the ESO MPI 2.2m telescope and the Suprime Cam on the Subaru $8.2 \mathrm{~m}$, allow us to identify the ICPNe associated with the extended ICL (Arnaboldi et al. 2002, 2003; Okamura et al. 2002; Aguerri et al. 2005). These surveys require the use of data reduction techniques suited for mosaic images, and also the development and refining of selection criteria based on color-magnitude diagrams from photometric catalogs, produced with SExtractor (Bertin \& Arnout 1996). 


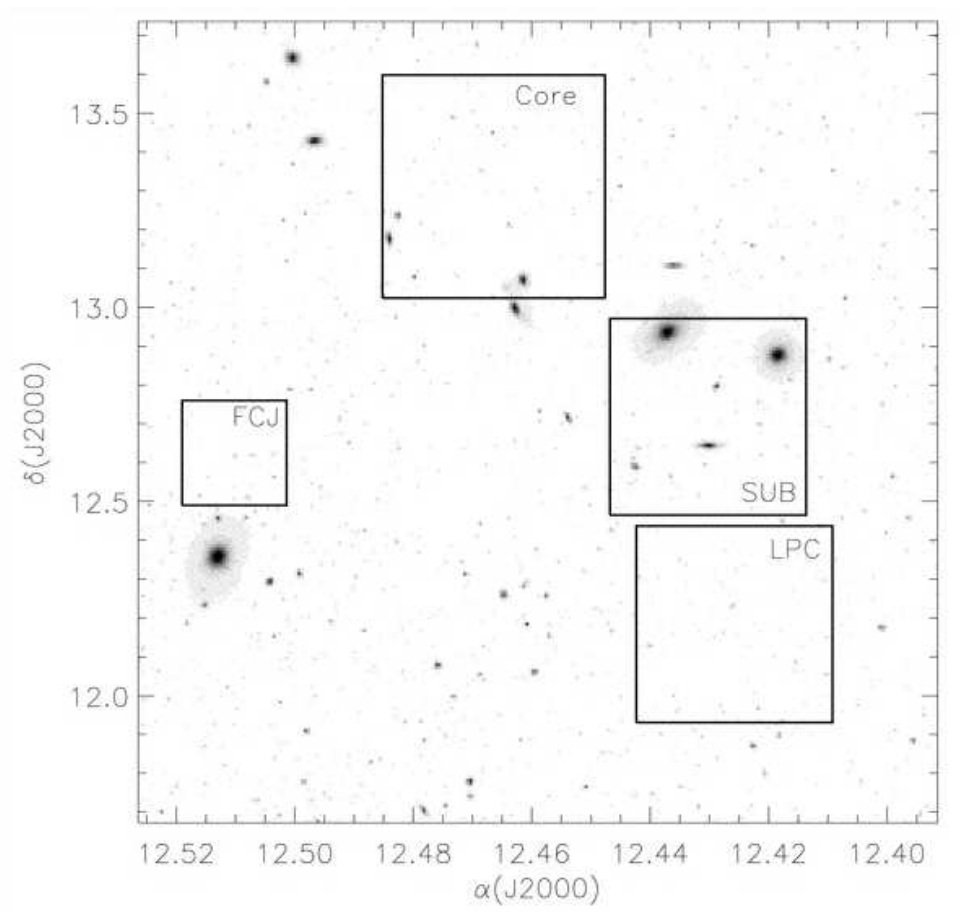

FIGURE 4. Aguerri et al. (2005) surveyed fields in the Virgo cluster core. The CORE field was obtained at the ESO MPI $2.2 \mathrm{~m}$ telescope, and the SUB field with the Suprime Cam at the $8.2 \mathrm{~m}$ Subaru telescope. The FCJ field is from prime focus camera of the Kitt Peak 4m telescope and the lower right field, LPC, was observed at the La Palma INT. From Aguerri et al. (2005).

The data analysed by Aguerri et al. (2005) constitute a sizable sample of ICPNe in the Virgo core region, constructed homogeneously and according to rigorous selection criteria; a layout of the pointings is shown in Figure 4 From the study of five wide-fields they conclude that the number density plot in Figure 5 shows no clear trend with distance from the cluster center at M87, except that the value in the innermost FCJ field is high. However, the spectroscopic results of Arnaboldi et al. (2004) have shown that $12 / 15 \mathrm{PNe}$ in this field have a low velocity dispersion of $250 \mathrm{kms}^{-1}$, i.e. in fact they belong to the outer halo of M87, which thus extends to at least $65 \mathrm{kpc}$ radius. In the SUB field, $8 / 13$ PNe belong to the similarly cold, extended halo of M84, while the remaining $\mathrm{PNe}$ are observed at velocities that are close to the systemic velocities of M86 and NGC 4388, the two other large galaxies in or near this field. It is possible that in a cluster as young and unrelaxed as Virgo, a substantial fraction of the ICL is still bound to the extended halos of galaxies, whereas in denser and older clusters these halos might already have been stripped. If so, it is not inappropriate to already count the luminosity in these halos as part of the ICL. However, in Figure 5 the plot of the PN number density with radius is also shown for the case in which the PNe in the outer halos of M87 and M84 are removed from the FCJ and SUB samples. In this case, the resulting number density is even more nearly flat with radius, but there are still significant field-to-field variations; in particular, the remaining number densities in SUB and LPC are low.

When one wishes to compare the luminosity of the ICL at the positions of Aguerri et al. (2005) fields with the luminosity from the Virgo galaxies, one adds in further uncertainties, because the luminosities of nearby Virgo galaxies depend very much on the location and field size surveyed in the Virgo Cluster. Aguerri et al. (2005) consider therefore their reported intervals in surface brightness to be their primary result, while the relative fractions of the ICL with respect to the Virgo galaxy light are evaluated for comparison with previous ICPN works, and considered them to be more uncertain.

From the study of four wide fields in the Virgo core, Aguerri et al. (2005) obtain a mean surface luminosity density of $2.7 \times 10^{6} \mathrm{~L}_{B \odot} \operatorname{arcmin}^{-2}, \mathrm{rms}=2.1 \times 10^{6}$ $\mathrm{L}_{B \odot} \operatorname{arcmin}^{-2}$, and a mean surface brightness of $\mu_{B}$ $=29.0 \mathrm{mag} \operatorname{arcsec}^{-2}$. Their best estimate of the ICL fractions with respect to light in galaxies in the Virgo core is $\sim 5 \%$. However, there are significant field-tofield variations. The fraction of the ICL versus total light ranges from $\sim 8 \%$ in the CORE and FCJ fields, to less than $1 \%$ in the LPC field, which in its low ICL fraction is similar to low-density environments (Castro-Rodríguez et al. 2003). This latter field corresponds to the lowest luminosity density in the mosaic image of the Virgo core 


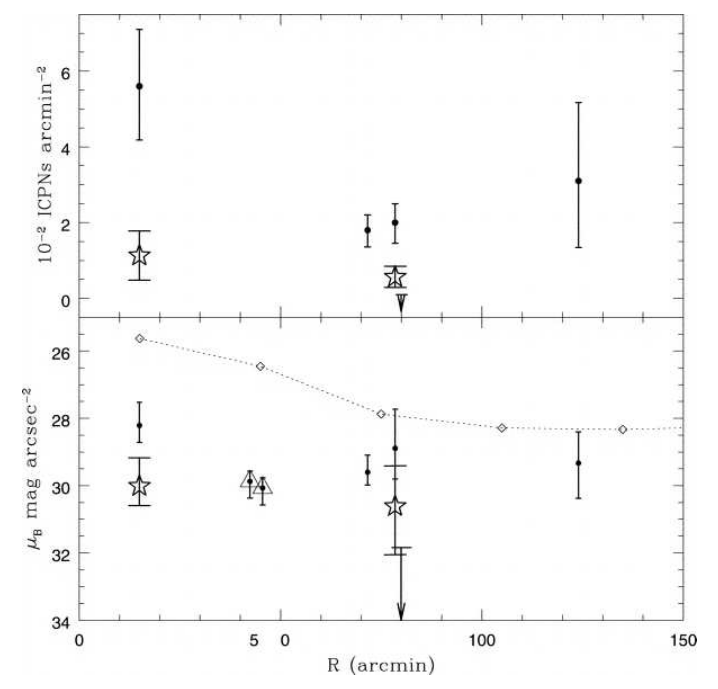

FIGURE 5. Number density of PNe (top) and surface brightness (bottom) in our surveyed fields. In the top panel, circles show the measured number densities from Table 3 of Aguerri et al. (2005), and error bars denote the Poisson errors. For the LPC field our upper limit is given. For the RCN1 field at the largest distance from M87, the uncertainty from the correction for $\operatorname{Ly} \alpha$ emitters is substantial and is included in the error bar. The large stars with Poisson error bars show the number densities of PNe in FCJ and SUB fields not including PNe bound to the halos of M87 and M84. In the lower panel, circles show the surface brightness inferred with the average value of $\alpha$ in Table 4 of Aguerri et al. (2005), and error bars show the range of values implied by the Poisson errors and the range of adopted $\alpha$ values. Triangles represent the measurements of the ICL from RGB stars; error bars indicate uncertainties in the metallicity, age, and distance of the parent population as discussed in Durrell et al. (2002). The stars indicate the surface brightness associated with the ICPNe in the FCJ and SUB fields that are not associated with the M87 or M84 halos but are free flying in the Virgo Cluster potential, (Arnaboldi et al. 2004). The dashed line and diamonds show the B-band luminosity of Virgo galaxies averaged in rings (Binggeli et al. 1987). Distances are relative to M87. The ICL shows no trend with cluster radius out to 150 arcmin. From Aguerri et al. (2005).

region from Mihos et al. (2005).

\section{SPECTROSCOPIC FOLLOW-UP}

ICPNe are the only component of the ICL whose kinematics can be measured at this time. This is important since the high-resolution N-body and hydrodynamical simulations predict that the ICL is un-relaxed, showing significant substructure in its spatial and velocity distributions in clusters similar to Virgo.

The spectroscopic follow-up with FLAMES of the ICPN candidates selected from three survey fields in the Virgo cluster core was carried out by Arnaboldi et al.
(2004). Radial velocities of 40 ICPNe in the Virgo cluster were obtained with the new multi-fiber FLAMES spectrograph on UT2 at VLT. The spectra were taken for a homogeneously selected sample of ICPNe, previously identified in three $\sim 0.25 \mathrm{deg}^{2}$ fields in the Virgo cluster core. For the first time, the $\lambda 4959 \AA$ line of the [OIII] doublet is seen in a large fraction (40\%) of ICPNe spectra, and a large fraction of the photometric candidates with $\mathrm{m}(5007)<27.2$ is spectroscopically confirmed.

\section{The LOS velocity distributions of ICPNe in the Virgo cluster core.}

With these data, Arnaboldi et al. (2004) were able for the first time to determine radial velocity distributions of ICPNe and use these to investigate the dynamical state of the Virgo cluster. Figure 6 shows an image of the Virgo cluster core with the positions of the imaged fields. The radial velocity distributions obtained from the FLAMES spectra in three of these fields are also displayed in Figure 6 Clearly the velocity distribution histograms for the three pointings are very different.

In the FCJ field, the ICPNe distribution is dominated by the halo of M87. There are 3 additional outliers, 2 at low velocity, which are also in the brightest PNLF bin, and therefore may be in front of the cluster. The surface brightness of the ICL associated with the 3 outliers, e.g. the ICPNe in the FCJ field, amounts to $\mu_{B} \simeq 30.63$ mag $\operatorname{arcsec}^{-2}$, in agreement with the surface brightness measurements of Ferguson et al. (1998) and Durrell et al. (2002) of the intracluster red giant stars.

The M87 peak of the FCJ velocity distribution contains 12 velocities with $\bar{v}_{p}=1276 \pm 71 \mathrm{~km} \mathrm{~s}^{-1}$ and $\sigma_{p}=247 \pm 52 \mathrm{~km} \mathrm{~s}^{-1}$. The average velocity is consistent with that of M87, $v_{\text {sys }}=1258 \mathrm{~km} \mathrm{~s}^{-1}$. The distance of the center of the FCJ field from the center of M87 is $15 .^{\prime} 0 \simeq 65 \mathrm{kpc}$ for an assumed M87 distance of $15 \mathrm{Mpc}$. The value of $\sigma_{p}$ is very consistent with the stellar velocity dispersion profile extrapolated outwards from $\simeq 150^{\prime \prime}$ in Figure 5 of Romanowsky \& Kochanek (2001) and falls in the range spanned by their dynamical models for the M87 stars. The main result from our measurement of $\sigma_{p}$ is that M87 has a stellar halo in approximate dynamical equilibrium out to at least $65 \mathrm{kpc}$.

In the CORE field, the distribution of ICPN LOS velocities is clearly broader than in the FCJ field. It has $\bar{v}_{C}=1491 \pm 290 \mathrm{~km} \mathrm{~s}^{-1}$ and $\sigma_{C}=1000 \pm 210 \mathrm{~km} \mathrm{~s}^{-1}$. The CORE field is in a region of Virgo devoid of bright galaxies, but contains 7 dwarfs, and 3 low luminosity E/S near its S/W borders. None of the confirmed ICPNe lies within a circle of three times half the major axis diameter of any of these galaxies, and there are no correlations of their velocities with the velocities of the nearest galaxies 
where these are known. Thus in this field there is a true IC stellar component.

The mean velocity of the ICPN in this field is consistent with that of 25 Virgo dE and dS0 within $2^{\circ}$ of M87, $<v_{\mathrm{dE}, \mathrm{M} 87}>=1436 \pm 108 \mathrm{~km} \mathrm{~s}^{-1}$ (Binggeli et al. 1987), and with that of $93 \mathrm{dE}$ and dS0 Virgo members, $<v_{\mathrm{dE} \text {,Virgo }}>=1139 \pm 67 \mathrm{~km} \mathrm{~s}^{-1}$ (Binggeli et al. 1993). However, the velocity dispersion of these galaxies is smaller, $\sigma_{\mathrm{dE}, \mathrm{M} 87}=538 \pm 77 \mathrm{~km} \mathrm{~s}^{-1}$ and $\sigma_{\mathrm{dE}, \text { Virgo }}=$ $649 \pm 48 \mathrm{~km} \mathrm{~s}^{-1}$.

The inferred luminosity from the ICPNe in the CORE field is $1.8 \times 10^{9} L_{B, \odot}$. This is about three times the luminosity of all dwarf galaxies in this field, $5.3 \times 10^{8} L_{B, \odot}$, but an order of magnitude less than the luminosities of the three low-luminosity E/S galaxies near the field borders. Using the results of Nulsen \& Böhringer (1995) and Matsushita et al. (2002), Arnaboldi et al. (2004) estimate the mass of the M87 subcluster inside $310 \mathrm{kpc}$ (the projected distance $D$ of the CORE field from M87) as $4.2 \times 10^{13} M_{\odot}$, and compute a tidal parameter $T$ for all these galaxies as the ratio of the mean density within the CORE field to the mean density of the galaxy. They find $T=0.01-0.06$, independent of galaxy luminosity. Since $T \sim D^{-2}$, any of these galaxies whose orbit now comes closer to M87 than $\sim 60 \mathrm{kpc}$ would be subject to severe tidal mass loss. Based on the evidence so far, a tantalizing possibility is that the ICPN population in the CORE field could be debris from the tidal disruption of small galaxies on nearby orbits in the M87 halo.

In the SUB field the velocity distribution from FLAMES spectra is again different from CORE and FCJ. The histogram of the LOS velocities shows substructures related to M86, M84 and NGC 4388, respectively, and in Figure 6 the projected phase space is shown. The association with the three galaxies is strengthened when we plot the LOS velocities of 4 HII regions (see Gerhard et al. 2002) detected with FLAMES in this pointing. The substructures in this distribution are highly correlated with the galaxy systemic velocities. The highest peak in the distribution coincides with M84, and even more so when we add the LOS velocities obtained previously at the TNG (Arnaboldi et al. 2003). The 10 TNG velocities give $\bar{v}_{\mathrm{M} 84}=1079 \pm 103 \mathrm{~km} \mathrm{~s}^{-1}$ and $\sigma_{\mathrm{M} 84}=325 \pm 75 \mathrm{~km}$ $\mathrm{s}^{-1}$ within a square of $4 R_{e} \times 4 R_{e}$ of the M84 center. The 8 FLAMES velocities give $\bar{v}_{\mathrm{M} 84}=891 \pm 74 \mathrm{~km} \mathrm{~s}^{-1}$ and $\sigma_{\mathrm{M} 84}=208 \pm 54 \mathrm{~km} \mathrm{~s}^{-1}$, going out to larger radii. Note that this includes the over-luminous PNe not attributed to M84 previously. The combined sample of 18 velocities gives $\bar{v}_{\mathrm{M} 84}=996 \pm 69 \mathrm{~km} \mathrm{~s}^{-1}$ and $\sigma_{\mathrm{M} 84}=293 \pm 50 \mathrm{~km}$ $\mathrm{s}^{-1}$. Most likely, all these PNe belong to a very extended halo around M84 (see the deep image in Arnaboldi et al. 1996). It is possible that the somewhat low velocity with respect to M84 may be a sign of tidal stripping by M86.

\section{FUTURE PROSPECTS AND CONCLUSIONS}

The observations indicate that the diffuse light is important in understanding cluster evolution, the star formation history and the enrichment of the Intracluster Medium. Measuring the projected phase space distribution of the IC stars constrains how and when this light originates, and the ICPNe are the only abundant stellar component of the ICL whose kinematics can be measured at this time.

These measurements are not restricted only to clusters within $25 \mathrm{Mpc}$ distance: by using a technique similar to those adopted for studies of $\operatorname{Ly} \alpha$ emitting galaxies at very high redshift, Gerhard et al. (2005) were able to detect $\mathrm{PNe}$ associated with the diffuse light in the Coma cluster, at $100 \mathrm{Mpc}$ distance, in a field which was previously studied by Bernstein et al. (1995); see also O. Gerhard's contribution, this conference. Now it has become possible to study ICL kinematics also in denser environments like the Coma cluster, and we can explore the effect of environments with different densities on galaxy evolution.

\section{ACKNOWLEDGMENTS}

M.A. would like to thank the organizing committee of the Conference on Planetary Nebulae as Astronomical Tools (Gdansk Poland, 28 June-2 July 2005) for the invitation to give this review. This work has been done in collaboration with Ortwin E. Gerhard, Kenneth C. Freeman, and J. Alfonso Aguerri, Massimo Capaccioli, Nieves Castro-Rodriguez, John Feldmeier, Fabio Governato, Rolf-P. Kudritzki, Roberto Mendez, Giuseppe Murante, Nicola R. Napolitano, Sadanori Okamura, Maurilio Pannella, Naoki Yasuda. M.A. wishes to thank ESO for the support of this project and the observing time allocated both at La Silla and Paranal Telescopes. M.A. wishes to thank the National Astronomical Observatory of Japan, for the observing time allocated at the Subaru Telescope. This work has been supported by INAF and the Swiss National Foundation.

\section{REFERENCES}

1. Aguerri, J.A.L. et al. 2005, AJ, 129, 2585

2. Adami, C. et al. 2005, A\&A, 429, 39

3. Arnaboldi, M. et al. 2004, ApJ, 614, L33

4. Arnaboldi, M. et al. 2003, AJ, 125, 514

5. Arnaboldi, M. et al. 2002, AJ, 123, 760

6. Arnaboldi, M. et al. 1996, ApJ, 472, 145

7. Bernstein, G.M., et al. 1995, AJ, 110, 1507

8. Bertin, E., \& Arnouts, S. 1996, A\&AS, 117, 39 


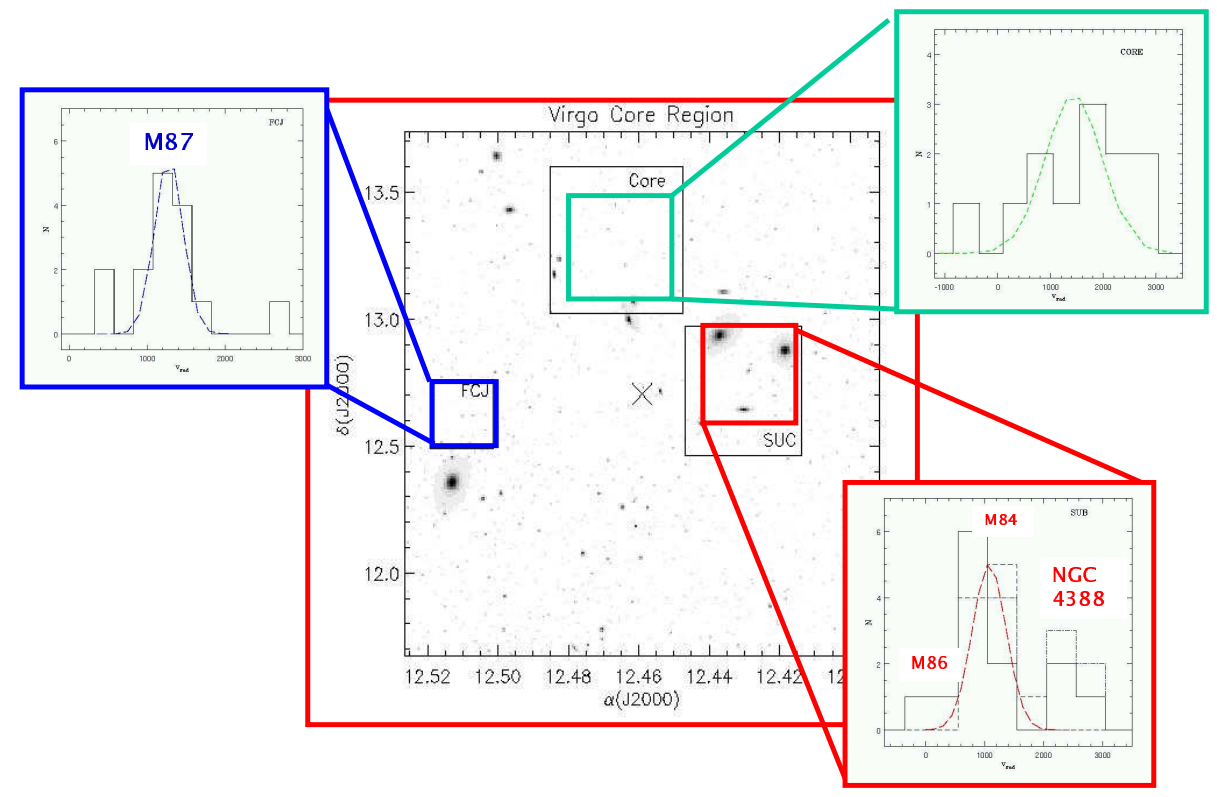

FIGURE 6. ICPN radial velocity distributions in the three pointings (FCJ, CORE, and SUB) from Aguerri et al. (2005). In FCJ panel, blue dashed line shows a Gaussian with $\bar{v}_{\text {rad }}=1276 \mathrm{~km} \mathrm{~s}^{-1}$ and $\sigma_{\text {rad }}=247 \mathrm{~km} \mathrm{~s}^{-1}$. In CORE, green dashed line shows a Gaussian with $\bar{v}_{\text {rad }}=1436 \mathrm{~km} \mathrm{~s}^{-1}$ and $\sigma_{\text {rad }}=538 \mathrm{~km} \mathrm{~s}^{-1}$, for VC galaxies dE and dS0 within $2^{\circ}$ of M87 (from Binggeli et al. 1987). In SUB, dashed histogram shows radial velocities from TNG spectroscopic follow-up (Arnaboldi et al. 2003). Dashed red line shows a Gaussian with $\bar{v}_{\text {rad }}=1080 \mathrm{~km} \mathrm{~s}^{-1}$ and $\sigma_{\text {rad }}=286 \mathrm{~km} \mathrm{~s}^{-1}$. Dashed-dotted lines show the SUB-FLAMES spectra including those spectra for HII regions, which have radial velocities in M84 \& NGC 4388 redshift ranges.

9. Binggeli, B., Popescu, C. C., \& Tammann, G. A., 1993, A\&A, 98, 275

10. Binggeli, B., Tammann, G.A., \& Sandage, A. 1987, AJ, 94, 251

11. Castro-Rodriguez, N. et al. 2003, A\&A, 405, 803

12. Dubinski, J. 1998, ApJ, 502, 141

13. Durrell, P.R. et al. 2002, ApJ, 570, 119

14. Feldmeier, J.J. et al. 2004a, ApJ, 615, 196

15. Feldmeier, J.J. et al. 2004b, in IAU Symp. 217, Recycling Intergalactic and Interstellar Matter, ed. P.-A. Duc, J. Braine, \& E. Brinks (San Francisco: ASP), 64

16. Feldmeier, J.J. et al. 2003, ApJS, 145, 65

17. Ferguson, H.C., Tanvir, N.R., \& von Hippel, T. 1998, Nature, 391, 461

18. Gal-Yam, A.H. et al. 2003, AJ, 125, 1087

19. Gerhard, O.E. et al. 2005, ApJ, 621, L93.

20. Gerhard, O.E., Arnaboldi, M., Freeman, K.C., \& Okamura, S. 2002, ApJ, 580, L121

21. Gonzalez, A. H., Zabludoff, A. I., Zaritsky, D., \& Dalcanton, J. J. 2000, ApJ, 536, 561

22. Gregg, M.D., \& West, M.J. 1998, Nature, 396, 549

23. Lin, Y-T, \& Mohr, J.J. 2004, ApJ, 617, 879

24. Malumuth, E. M., \& Richstone, D. O. 1984, ApJ, 276, 413

25. Matsushita, K., Belsole, E., Finoguenov, A., \& Böhringer, H. 2002, A\&A, 386, 77

26. Merritt, D. 1983, ApJ, 264, 24
27. Merritt, D. 1984, ApJ, 276, 26

28. Mihos, C. et al. 2005, ApJL, in press (astro-ph/0508217)

29. Miller, G. E. 1983, ApJ, 268, 495

30. Moore, B. et al. 1996, Nature, 379, 613

31. Murante, G. et al. 2004, ApJ, 607, L83

32. Napolitano, N.R. et al. 2003, ApJ, 594, 172

33. Nulsen, P. E. J., \& Böhringer, H., 1995, MNRAS, 274, 1093

34. Oemler, A. 1973, ApJ, 180, 11

35. Okamura, S. et al. 2002, PASJ, 54, 883

36. Richstone, D. O., \& Malumuth, E. M. 1983, ApJ, 268, 30

37. Romanowsky, A. J., \& Kochanek, C. S. 2001, ApJ, 553, 722

38. Schombert, J. 1986, ApJS, 60, 603

39. Sommer-Larsen, J., Romeo, A.D. \& Portinari, L. 2005, MNRAS, 357, 478

40. Theuns, T., \& Warren, S. J. 1997, MNRAS, 284, L11

41. Thuan, T. X., \& Kormendy, J. 1977, PASP, 89, 466

42. Willman, B. et al. 2004, MNRAS, 355, 159

43. Zibetti, S. et al. 2005, MNRAS, 358, 949

44. Zwicky, F., 1951, PASP, 63, 61 